Review Article

\title{
Pneumomediastinum with COVID-19: a natural process or complication? A literature review
}

\author{
Elmutaz Kanani* \\ Department of General Surgery, Khoula Hospital, Muscat, Oman \\ Received: 16 August 2020 \\ Accepted: 07 October 2020 \\ *Correspondence: \\ Dr. Elmutaz Kanani, \\ E-mail: mutazkanani313@hotmail.com \\ Copyright: ( $)$ the author(s), publisher and licensee Medip Academy. This is an open-access article distributed under \\ the terms of the Creative Commons Attribution Non-Commercial License, which permits unrestricted non-commercial \\ use, distribution, and reproduction in any medium, provided the original work is properly cited.
}

\begin{abstract}
COVID-19 pneumonia is a serious respiratory viral infection that recently spread all over the world. It carries a significant mortality risk. Little is known about the pathophysiology of the disease, being a newly mutated virus. Knowledge of the clinical disease manifestations kept evolving as unfamiliar findings and presentations are constantly reported. Pneumomediastinum had been frequently reported since the start of the pandemic, and its significance was questioned. The aim of this review is to analyze the reported cases of pneumomediastinum with coronavirus infection to look for its pathophysiology, prognostic value, and best treatment. A systematic literature search was conducted using free text search for pneumomediastinum with COVID-19 case reports and case series. PubMed and Google scholar data bases were searched. Twenty three articles were retrieved. All are case reports and case series, 39 cases of the reported cohort qualified for inclusion into analysis. Chronic respiratory conditions were found in $23 \%$ of the patients. There was about four folds risk of developing the condition with oxygen therapy (both through invasive and non-invasive means). The best diagnostic modality was chest computed tomography scan (74.4\%). Management was conservative in $69 \%$, while pleural drainage was necessary in $23 \%$ of the patients. Both treatment strategies were successful with no statistical difference ( $\mathrm{p}$ value=0.327). It is not yet clear if pneumomediastinum is a pathological disease process or an interventional complication. Interventional and conservative treatment had similar outcomes depending on individual case merits. No prognostic value could be demonstrated.
\end{abstract}

Keywords: COVID-19, Emphysema, Pneumomediastinum

\section{INTRODUCTION}

Coronavirus disease (COVID 19) is a serious lifethreatening disease with global spread. As per the World Health Organization (WHO); more than 18 million cases had been reported by the first week of August 2020. Fatalities were reported to exceed seven hundred thousand for the same period.

Healthcare systems were overwhelmed by the influx of huge numbers of patients requiring medical care with high demand for critical care and mechanical ventilation. The disease had negatively impacted the economies and public activity. Nothing had united the whole world recently like the COVID-19 pandemic. The world had shared the same interests and fears. Comparable preventive measures were rehearsed in all edges of the world. This can't be professed to be the case in the medical and scientific community. There is as yet an ongoing debate about the best treatment strategy. There is no consensus to date about the range of the disease manifestations and newly recognized symptoms are being reported every now and then.

Pneumomediastinum is one of these emerging findings. It is not a common condition, and it is said to be present when free air is present within the mediastinum. This can be caused by numerous intra and extra-thoracic pathologies that lead to air leak into the mediastinum. ${ }^{1}$ 
The condition had been reported recently from different parts of the world with conflicting explanations whether it is part of the disease process or a complication. An argument about its prognostic implications was also reflected.

In this review these individual case reports were analyzed in an attempt to come up with a conclusion from the available evidence.

\section{Related anatomy and pathophysiology}

The mediastinum is the space sandwiched between the two pleural cavities, extending from the thoracic inlet above to the diaphragm below and bounded by the sternum anteriorly and the vertebral column posteriorly. ${ }^{1}$ It communicates with many anatomic spaces including: submandibular space, retropharyngeal space, vascular sheaths in the neck, retroperitoneum, flanks, and pelvis. ${ }^{1}$

Although the pneumomediastinum was first described by Laenek in 1827, the explanation of its pathophysiology was provided later in 1944 by Macklin and Macklin. ${ }^{2}$ They concluded that alveolar rupture results from increased intra-alveolar pressure. The resultant leaked air migrates through the peribronchial and perivascular sheaths into the mediastinum. ${ }^{2}$ The leaked air may dissect through the loose areolar tissue into the neck and subcutaneous space, and into the peritoneal cavity resulting in subcutaneous emphysema and pneumoperitoneum, respectively. It may also result in pneumothorax. ${ }^{2}$

\section{METHODS}

A PubMed and Google scholar free text search was conducted between the 3rd and 8th of August 2020 using the term "pneumomediastinum in COVID-19". It yielded 23 related articles in English language. All the articles are case reports and case series.

All reported confirmed COVID-19 positive patients who developed pneumomediastinum were included in the study, whether they had received invasive or non-invasive oxygen therapy.

All the reported cases were analyzed for age, gender, comorbidities, presence of cough as a presenting symptom, mode of oxygen therapy, time of pneumomediastinum diagnosis in relation to endo-tracheal intubation, the diagnostic modality, presence or absence of airway injury, management, and the final outcome.

Microsoft Office Excel (2007) was used for the statistical analysis. $P$ value $<0.05$ was considered significant.

\section{RESULTS}

A total of twenty three articles were deemed relative to the topic of interest and subjected to analysis. All are case reports and case series. Inclusion criteria were met by 39 cases, and they were subjected to the analysis. Table 1 summarizes all the reported coronavirus infections with pneumomediastinum cases.

Table 1: Summary of coronavirus infection with pneumomediastinum reported cases.

\begin{tabular}{|c|c|c|c|c|c|c|c|c|c|c|}
\hline $\begin{array}{l}\text { Patie } \\
\text {-nt }\end{array}$ & $\begin{array}{l}\text { Age } \\
\text { (years) }\end{array}$ & Sex & Co-morbidities & $\begin{array}{l}\text { Co- } \\
\text { ugh }\end{array}$ & $\begin{array}{l}\text { Mode } \\
\text { of } \mathrm{O}_{2} \\
\text { therap } \\
-\mathrm{y}\end{array}$ & $\begin{array}{l}\text { Time of } \\
\text { PTX/PM } \\
\text { diagnosi } \\
\text { s related } \\
\text { to } \\
\text { intuba- } \\
\text { tion }\end{array}$ & $\begin{array}{l}\text { Diagnost } \\
\text {-ic } \\
\text { modality }\end{array}$ & $\begin{array}{l}\text { Air } \\
- \\
\text { way } \\
\text { inju } \\
\text {-ry }\end{array}$ & $\begin{array}{l}\text { Manag- } \\
\text { ement }\end{array}$ & $\begin{array}{l}\text { Outc- } \\
\text { ome }\end{array}$ \\
\hline $\mathbf{1}^{3}$ & 84 & $\mathrm{~F}$ & $\begin{array}{l}\text { Hypertension, } \\
\text { hypercholesterolemia, } \\
\text { heart failure, prosthetic } \\
\text { valve replacement, renal } \\
\text { failure }\end{array}$ & Yes & $\begin{array}{l}\text { Non- } \\
\text { invasiv } \\
\text {-e }\end{array}$ & $\begin{array}{l}\text { Not } \\
\text { intubated }\end{array}$ & $\begin{array}{l}\text { Chest CT } \\
\text { (PM, } \\
\text { PTX) }\end{array}$ & - & N/A & Died \\
\hline $2^{3}$ & 67 & M & Nil & No & $\begin{array}{l}\text { Non- } \\
\text { invasiv } \\
\text {-e }\end{array}$ & $\begin{array}{l}\text { Not } \\
\text { intubated }\end{array}$ & $\begin{array}{l}\text { CXR } \\
(\mathrm{PM}, \\
\text { PTX) }\end{array}$ & - & ICD & Died \\
\hline $3^{3}$ & 73 & M & $\begin{array}{l}\text { Basal cell epithelioma, } \\
\text { obstructive sleep apnoea, } \\
\text { obesity, depression }\end{array}$ & No & $\begin{array}{l}\text { Non- } \\
\text { invasiv } \\
\text {-e }\end{array}$ & $\begin{array}{l}\text { Not } \\
\text { intubated }\end{array}$ & $\begin{array}{l}\text { Chest CT } \\
(\mathrm{PM})\end{array}$ & - & $\begin{array}{l}\text { Conser- } \\
\text { vative }\end{array}$ & Died \\
\hline $4^{4}$ & 38 & M & Nil & Yes & $\begin{array}{l}\text { Non- } \\
\text { invasiv } \\
\text {-e }\end{array}$ & $\begin{array}{l}\text { Not } \\
\text { intubated }\end{array}$ & $\begin{array}{l}\text { Chest CT } \\
(\mathrm{PM}, \mathrm{SE})\end{array}$ & - & $\begin{array}{l}\text { Conser- } \\
\text { vative }\end{array}$ & $\begin{array}{l}\text { Resol } \\
\text {-ved }\end{array}$ \\
\hline $5^{5}$ & 62 & M & Nil & Yes & $\begin{array}{l}\text { Non- } \\
\text { invasiv } \\
\text {-e }\end{array}$ & $\begin{array}{l}\text { Not } \\
\text { intubated }\end{array}$ & $\begin{array}{l}\text { Chest CT } \\
\text { (PM, } \\
\text { PTX, } \\
\text { SE) }\end{array}$ & - & $\begin{array}{l}\text { Conser- } \\
\text { vative }\end{array}$ & $\begin{array}{l}\text { Resol } \\
\text {-ved }\end{array}$ \\
\hline
\end{tabular}




\begin{tabular}{|c|c|c|c|c|c|c|c|c|c|c|}
\hline $\begin{array}{l}\text { Patie } \\
-n t\end{array}$ & $\begin{array}{l}\text { Age } \\
\text { (years) }\end{array}$ & Sex & Co-morbidities & $\begin{array}{l}\text { Co- } \\
\text { ugh }\end{array}$ & $\begin{array}{l}\text { Mode } \\
\text { of } \mathrm{O}_{2} \\
\text { therap } \\
-\mathrm{y}\end{array}$ & $\begin{array}{l}\text { Time of } \\
\text { PTX/PM } \\
\text { diagnosi } \\
\text { s related } \\
\text { to } \\
\text { intuba- } \\
\text { tion }\end{array}$ & $\begin{array}{l}\text { Diagnost } \\
\text {-ic } \\
\text { modality }\end{array}$ & $\begin{array}{l}\text { Air } \\
- \\
\text { way } \\
\text { inju } \\
\text {-ry }\end{array}$ & $\begin{array}{l}\text { Manag- } \\
\text { ement }\end{array}$ & $\begin{array}{l}\text { Outc- } \\
\text { ome }\end{array}$ \\
\hline $6^{6}$ & 67 & M & $\begin{array}{l}\text { Coronary artery bypass, } \\
\text { chronic pulmonary } \\
\text { diseases including } \\
\text { obsolete pulmonary } \\
\text { tuberculosis, chronic } \\
\text { bronchitis, and } \\
\text { emphysema }\end{array}$ & No & $\begin{array}{l}\text { Invasiv } \\
\text {-e }\end{array}$ & $\begin{array}{l}\text { After } \\
\text { Intuba- } \\
\text { tion }\end{array}$ & $\begin{array}{l}\text { CXR } \\
\text { (PM, } \\
\text { PTX, } \\
\text { SE) }\end{array}$ & No & ICD & Died \\
\hline $7^{7}$ & 49 & M & Hypertension, NIDDM & Yes & $\begin{array}{l}\text { Non- } \\
\text { invasiv } \\
\text {-e }\end{array}$ & $\begin{array}{l}\text { Not } \\
\text { intubated }\end{array}$ & $\begin{array}{l}\text { Chest CT } \\
(\mathrm{PM}, \mathrm{SE})\end{array}$ & No & $\begin{array}{l}\text { Conser- } \\
\text { vative }\end{array}$ & $\begin{array}{l}\text { Resol } \\
\text {-ved }\end{array}$ \\
\hline $8^{8}$ & 57 & M & Nil & Yes & $\begin{array}{l}\text { Invasiv } \\
\text {-e }\end{array}$ & $\begin{array}{l}\text { After } \\
\text { intuba- } \\
\text { tion }\end{array}$ & $\begin{array}{l}\text { Chest CT } \\
(\mathrm{PM}, \mathrm{SE})\end{array}$ & No & N/A & N/A \\
\hline $9^{9}$ & - & - & $\begin{array}{l}\text { Lung disease: } \\
\text { emphysema, smoker }\end{array}$ & No & $\begin{array}{l}\text { Non- } \\
\text { invasiv } \\
\text {-e }\end{array}$ & $\begin{array}{l}\text { Not } \\
\text { intubated }\end{array}$ & $\begin{array}{l}\text { Chest CT } \\
(\mathrm{PM}, \mathrm{SE})\end{array}$ & - & $\begin{array}{l}\text { Conser- } \\
\text { vative }\end{array}$ & $\begin{array}{l}\text { Resol } \\
\text {-ved }\end{array}$ \\
\hline $10^{9}$ & - & - & - & Yes & $\begin{array}{l}\text { Non- } \\
\text { invasiv } \\
\text {-e }\end{array}$ & $\begin{array}{l}\text { Before } \\
\text { intuba- } \\
\text { tion }\end{array}$ & $\begin{array}{l}\text { Chest CT } \\
(\mathrm{PM}, \mathrm{SE})\end{array}$ & - & $\begin{array}{l}\text { Conser- } \\
\text { vative }\end{array}$ & $\begin{array}{l}\text { Persis } \\
\text {-ted }\end{array}$ \\
\hline $11^{9}$ & - & - & Asthma & Yes & $\begin{array}{l}\text { Non- } \\
\text { invasiv } \\
\text {-e }\end{array}$ & $\begin{array}{l}\text { Before } \\
\text { intuba- } \\
\text { tion }\end{array}$ & $\begin{array}{l}\text { Chest CT } \\
(\mathrm{PM}, \mathrm{SE})\end{array}$ & No & $\begin{array}{l}\text { Conser- } \\
\text { vative }\end{array}$ & $\begin{array}{l}\text { Resol } \\
\text {-ved }\end{array}$ \\
\hline $12^{9}$ & - & - & Nil & Yes & $\begin{array}{l}\text { Non- } \\
\text { invasiv } \\
\mathrm{e}\end{array}$ & $\begin{array}{l}\text { Not } \\
\text { intubated }\end{array}$ & $\begin{array}{l}\text { Chest CT } \\
\text { (PM, } \\
\text { PTX) }\end{array}$ & - & ICD & $\begin{array}{l}\text { Resol } \\
\text {-ved }\end{array}$ \\
\hline $13^{9}$ & - & - & Asthma & Yes & $\begin{array}{l}\text { Non- } \\
\text { invasiv } \\
\text {-e }\end{array}$ & $\begin{array}{l}\text { Not } \\
\text { intubated }\end{array}$ & $\begin{array}{l}\text { Chest CT } \\
(\mathrm{PM}, \mathrm{SE})\end{array}$ & - & $\begin{array}{l}\text { Conser- } \\
\text { vative }\end{array}$ & $\begin{array}{l}\text { Persis } \\
\text {-ted }\end{array}$ \\
\hline $14^{10}$ & 59 & $\mathrm{~F}$ & Morbid obesity & No & $\begin{array}{l}\text { Invasiv } \\
\text {-e }\end{array}$ & $\begin{array}{l}\text { During } \\
\text { intuba- } \\
\text { tion }\end{array}$ & $\begin{array}{l}\text { Chest CT } \\
\text { (SE, PM, } \\
\text { PTX) }\end{array}$ & Yes & $\begin{array}{l}\text { Surgi-cal } \\
\text { repair }\end{array}$ & N/A \\
\hline $15^{10}$ & 67 & M & Morbid obesity & No & $\begin{array}{l}\text { Invasiv } \\
-\mathrm{e}\end{array}$ & $\begin{array}{l}\text { After } \\
\text { intuba- } \\
\text { tion }\end{array}$ & $\begin{array}{l}\text { Chest CT } \\
\text { (PM, } \\
\text { PTX) }\end{array}$ & Yes & $\begin{array}{l}\text { Conser- } \\
\text { vative }\end{array}$ & N/A \\
\hline $16^{11}$ & 36 & M & IDDM & Yes & $\begin{array}{l}\text { Non- } \\
\text { invasiv } \\
\text {-e }\end{array}$ & $\begin{array}{l}\text { Before } \\
\text { intuba- } \\
\text { tion }\end{array}$ & $\begin{array}{l}\text { CXR } \\
(\mathrm{PM}, \mathrm{SE})\end{array}$ & No & $\begin{array}{l}\text { Conser- } \\
\text { vative }\end{array}$ & $\begin{array}{l}\text { Resol } \\
\text { ved }\end{array}$ \\
\hline $17^{11}$ & 47 & M & Drug and alcohol abuse & No & $\begin{array}{l}\text { Invasiv } \\
\text {-e }\end{array}$ & $\begin{array}{l}\text { After } \\
\text { intuba- } \\
\text { tion }\end{array}$ & $\begin{array}{l}\text { CXR } \\
(\mathrm{PM}, \mathrm{SE})\end{array}$ & No & $\begin{array}{l}\text { Conser- } \\
\text { vative }\end{array}$ & $\begin{array}{l}\text { Resol } \\
\text { ved }\end{array}$ \\
\hline $18^{11}$ & 78 & M & $\begin{array}{l}\text { Hypertension, } \\
\text { hyperlipidemia }\end{array}$ & No & $\begin{array}{l}\text { Invasiv } \\
\text {-e }\end{array}$ & $\begin{array}{l}\text { After } \\
\text { intuba- } \\
\text { tion }\end{array}$ & $\begin{array}{l}\text { CXR } \\
(\mathrm{PM}, \mathrm{SE})\end{array}$ & No & $\begin{array}{l}\text { Conser- } \\
\text { vative }\end{array}$ & Died \\
\hline $19^{12}$ & 77 & M & Nil & No & $\begin{array}{l}\text { Invasiv } \\
\text {-e }\end{array}$ & $\begin{array}{l}\text { After } \\
\text { intuba- } \\
\text { tion }\end{array}$ & $\begin{array}{l}\text { Chest CT } \\
(\mathrm{PM}, \mathrm{SE})\end{array}$ & No & $\begin{array}{l}\text { Conser- } \\
\text { vative }\end{array}$ & N/A \\
\hline $20^{13}$ & 44 & M & Nil & Yes & $\begin{array}{l}\text { Non- } \\
\text { invasiv } \\
\text {-e }\end{array}$ & $\begin{array}{l}\text { Not } \\
\text { intubated }\end{array}$ & $\begin{array}{l}\text { Chest CT } \\
(\mathrm{PM})\end{array}$ & No & $\begin{array}{l}\text { Conser- } \\
\text { vative }\end{array}$ & $\begin{array}{l}\text { Resol } \\
\text {-ved }\end{array}$ \\
\hline $21^{14}$ & 64 & M & $\begin{array}{l}\text { NIDDM, obstructive } \\
\text { sleep apnoea }\end{array}$ & N/A & $\begin{array}{l}\text { Non- } \\
\text { invasiv } \\
\text {-e }\end{array}$ & $\begin{array}{l}\text { Not } \\
\text { intubated }\end{array}$ & $\begin{array}{l}\text { Chest CT } \\
(\mathrm{PM})\end{array}$ & No & $\begin{array}{l}\text { Conser- } \\
\text { vative }\end{array}$ & N/A \\
\hline
\end{tabular}




\begin{tabular}{|c|c|c|c|c|c|c|c|c|c|c|}
\hline $\begin{array}{l}\text { Patie } \\
-n t\end{array}$ & $\begin{array}{l}\text { Age } \\
\text { (years) }\end{array}$ & Sex & Co-morbidities & $\begin{array}{l}\text { Co- } \\
\text { ugh }\end{array}$ & $\begin{array}{l}\text { Mode } \\
\text { of } \mathrm{O}_{2} \\
\text { therap } \\
-\mathrm{y}\end{array}$ & $\begin{array}{l}\text { Time of } \\
\text { PTX/PM } \\
\text { diagnosi } \\
\text { s related } \\
\text { to } \\
\text { intuba- } \\
\text { tion }\end{array}$ & $\begin{array}{l}\text { Diagnost } \\
\text {-ic } \\
\text { modality }\end{array}$ & $\begin{array}{l}\text { Air } \\
- \\
\text { way } \\
\text { inju } \\
\text {-ry }\end{array}$ & $\begin{array}{l}\text { Manag- } \\
\text { ement }\end{array}$ & $\begin{array}{l}\text { Outc- } \\
\text { ome }\end{array}$ \\
\hline $22^{15}$ & 38 & M & Nil & Yes & $\begin{array}{l}\text { Non- } \\
\text { invasiv } \\
\text {-e }\end{array}$ & $\begin{array}{l}\text { Not } \\
\text { intubated }\end{array}$ & $\begin{array}{l}\text { Chest CT } \\
\text { (PM, } \\
\text { PTX) }\end{array}$ & No & $\begin{array}{l}\text { Conser- } \\
\text { vative }\end{array}$ & $\begin{array}{l}\text { Resol } \\
\text {-ved }\end{array}$ \\
\hline $23^{16}$ & 36 & $\mathrm{~F}$ & Nil & Yes & $\begin{array}{l}\text { Non- } \\
\text { invasiv } \\
\text {-e }\end{array}$ & $\begin{array}{l}\text { Not } \\
\text { intubated }\end{array}$ & $\begin{array}{l}\text { Chest CT } \\
(\mathrm{PM})\end{array}$ & No & $\begin{array}{l}\text { Conser- } \\
\text { vative }\end{array}$ & Died \\
\hline $24^{17}$ & 23 & $\mathrm{~F}$ & Nil & No & No & $\begin{array}{l}\text { Not } \\
\text { intubated }\end{array}$ & $\begin{array}{l}\text { Chest CT } \\
(\mathrm{PM})\end{array}$ & No & $\begin{array}{l}\text { Conser- } \\
\text { vative }\end{array}$ & $\begin{array}{c}\text { Resol } \\
\text { Continued }\end{array}$ \\
\hline $25^{18}$ & 78 & $\mathrm{~F}$ & DM, hypertension & Yes & No & $\begin{array}{l}\text { Not } \\
\text { intubated }\end{array}$ & $\begin{array}{l}\text { Chest CT } \\
(\mathrm{PM})\end{array}$ & No & $\begin{array}{l}\text { Conser- } \\
\text { vative }\end{array}$ & $\begin{array}{l}\text { Resol } \\
\text {-ved }\end{array}$ \\
\hline $26^{18}$ & 41 & M & Nil & No & No & $\begin{array}{l}\text { Not } \\
\text { intubated }\end{array}$ & $\begin{array}{l}\text { Chest CT } \\
\text { (PM, } \\
\text { PTX, } \\
\text { SE) }\end{array}$ & No & ICD & $\begin{array}{l}\text { Resol } \\
\text {-ved }\end{array}$ \\
\hline $27^{19}$ & 30 & M & N/A & No & No & $\begin{array}{l}\text { Not } \\
\text { intubated }\end{array}$ & $\begin{array}{l}\text { Chest CT } \\
(\mathrm{PM}, \mathrm{SE})\end{array}$ & No & $\begin{array}{l}\text { Conser- } \\
\text { vative }\end{array}$ & $\begin{array}{l}\text { Resol } \\
\text {-ved }\end{array}$ \\
\hline $28^{19}$ & 65 & M & N/A & No & No & $\begin{array}{l}\text { Not } \\
\text { intubated }\end{array}$ & $\begin{array}{l}\text { Chest CT } \\
(\mathrm{PM}, \mathrm{SE})\end{array}$ & No & $\begin{array}{l}\text { Conser- } \\
\text { vative }\end{array}$ & $\begin{array}{l}\text { Resol } \\
\text {-ved }\end{array}$ \\
\hline $29^{20}$ & 55 & $\mathrm{~F}$ & $\begin{array}{l}\text { Hypertension, asthma, } \\
\text { morbid obesity }\end{array}$ & Yes & $\begin{array}{l}\text { Non- } \\
\text { invasiv } \\
\text {-e }\end{array}$ & $\begin{array}{l}\text { Not } \\
\text { intubated }\end{array}$ & $\begin{array}{l}\text { Chest CT } \\
\text { (PM, } \\
\text { PTX) }\end{array}$ & No & $\begin{array}{l}\text { Conser- } \\
\text { vative }\end{array}$ & Died \\
\hline $3^{20}$ & 31 & M & $\begin{array}{l}\text { Chronic gastritis, } \\
\text { hypercholesterolemia }\end{array}$ & Yes & $\begin{array}{l}\text { Non- } \\
\text { invasiv } \\
\text {-e }\end{array}$ & $\begin{array}{l}\text { Not } \\
\text { intubated }\end{array}$ & $\begin{array}{l}\text { Chest CT } \\
(\mathrm{PM})\end{array}$ & No & $\begin{array}{l}\text { Conser- } \\
\text { vative }\end{array}$ & $\begin{array}{l}\text { Resol } \\
\text {-ved }\end{array}$ \\
\hline $31^{21}$ & 70 & M & Hypothyroidism, smoker & Yes & $\begin{array}{l}\text { Invasiv } \\
\text {-e }\end{array}$ & $\begin{array}{l}\text { After } \\
\text { intuba- } \\
\text { tion }\end{array}$ & $\begin{array}{l}\text { Chest CT } \\
(\mathrm{PM}, \mathrm{SE})\end{array}$ & No & $\begin{array}{l}\text { Bilateral } \\
\text { ICDs, } \\
\text { bilateral } \\
\text { subcutan } \\
\text {-eous } \\
\text { drains }\end{array}$ & $\begin{array}{l}\text { Resol } \\
\text {-ved }\end{array}$ \\
\hline $32^{21}$ & 60 & M & IDDM, pancreatitis & No & $\begin{array}{l}\text { Invasiv } \\
\text {-e }\end{array}$ & $\begin{array}{l}\text { After } \\
\text { intuba- } \\
\text { tion }\end{array}$ & $\begin{array}{l}\text { Chest CT } \\
(\mathrm{PM}, \mathrm{SE})\end{array}$ & Yes & $\begin{array}{l}\text { Bilateral } \\
\text { ICDs, } \\
\text { bilateral } \\
\text { subcutan } \\
\text {-eous } \\
\text { drains }\end{array}$ & $\begin{array}{l}\text { Resol } \\
\text {-ved }\end{array}$ \\
\hline $33^{21}$ & 38 & M & Nil & Yes & $\begin{array}{l}\text { Invasiv } \\
\text {-e }\end{array}$ & $\begin{array}{l}\text { After } \\
\text { intuba- } \\
\text { tion }\end{array}$ & $\begin{array}{l}\text { CXR } \\
(\mathrm{PM}, \mathrm{SE})\end{array}$ & N/A & $\begin{array}{l}\text { Bilateral } \\
\text { ICDs }\end{array}$ & Died \\
\hline $34^{21}$ & 51 & M & Asthma & Yes & $\begin{array}{l}\text { Invasiv } \\
\text {-e }\end{array}$ & $\begin{array}{l}\text { After } \\
\text { intuba- } \\
\text { tion }\end{array}$ & $\begin{array}{l}\text { CXR } \\
\text { (PM, } \\
\text { PTX) }\end{array}$ & N/A & Left ICD & Died \\
\hline $35^{21}$ & 60 & M & Early prostate cancer & No & $\begin{array}{l}\text { Invasiv } \\
\text {-e }\end{array}$ & $\begin{array}{l}\text { After } \\
\text { intuba- } \\
\text { tion }\end{array}$ & $\begin{array}{l}\text { CXR } \\
(\mathrm{PM}, \mathrm{SE})\end{array}$ & No & $\begin{array}{l}\text { Conserva } \\
\text {-tive }\end{array}$ & $\begin{array}{l}\text { Resol } \\
\text {-ved }\end{array}$ \\
\hline $36^{22}$ & 52 & $\mathrm{M}$ & Nil & Yes & No & $\begin{array}{l}\text { Before } \\
\text { intuba- } \\
\text { tion }\end{array}$ & $\begin{array}{l}\text { Chest CT } \\
(\mathrm{PM})\end{array}$ & No & $\begin{array}{l}\text { Conserva } \\
\text {-tive }\end{array}$ & $\begin{array}{l}\text { Resol } \\
\text {-ved }\end{array}$ \\
\hline $37^{23}$ & 52 & M & DM, asthma & Yes & $\begin{array}{l}\text { Non- } \\
\text { invasiv } \\
\text {-e }\end{array}$ & $\begin{array}{l}\text { After } \\
\text { intuba- } \\
\text { tion }\end{array}$ & $\begin{array}{l}\text { CXR } \\
(\mathrm{PM})\end{array}$ & No & $\begin{array}{l}\text { Conserva } \\
\text {-tive }\end{array}$ & $\begin{array}{l}\text { Resol } \\
\text {-ved }\end{array}$ \\
\hline
\end{tabular}

Continued. 


\begin{tabular}{|c|c|c|c|c|c|c|c|c|c|c|}
\hline $\begin{array}{l}\text { Patie } \\
-n t\end{array}$ & $\begin{array}{l}\text { Age } \\
\text { (years) }\end{array}$ & Sex & Co-morbidities & $\begin{array}{l}\text { Co- } \\
\text { ugh }\end{array}$ & $\begin{array}{l}\text { Mode } \\
\text { of } \mathrm{O}_{2} \\
\text { therap } \\
-\mathrm{y}\end{array}$ & $\begin{array}{l}\text { Time of } \\
\text { PTX/PM } \\
\text { diagnosi } \\
\text { s related } \\
\text { to } \\
\text { intuba- } \\
\text { tion }\end{array}$ & $\begin{array}{l}\text { Diagnost } \\
\text {-ic } \\
\text { modality }\end{array}$ & $\begin{array}{l}\text { Air } \\
- \\
\text { way } \\
\text { inju } \\
\text {-ry }\end{array}$ & $\begin{array}{l}\text { Manag- } \\
\text { ement }\end{array}$ & $\begin{array}{l}\text { Outc- } \\
\text { ome }\end{array}$ \\
\hline $38^{23}$ & 68 & M & $\begin{array}{l}\text { Hypertension, } \\
\text { hypercholesterolemia }\end{array}$ & Yes & $\begin{array}{l}\text { Invasiv } \\
\text {-e }\end{array}$ & $\begin{array}{l}\text { After } \\
\text { intuba- } \\
\text { tion }\end{array}$ & $\begin{array}{l}\text { Chest CT } \\
\text { (PM, } \\
\text { PTX, } \\
\text { SE) }\end{array}$ & No & ICD & $\begin{array}{l}\text { Resol } \\
\text {-ved }\end{array}$ \\
\hline $39^{23}$ & 66 & M & $\begin{array}{l}\text { Hypertension, obesity, } \\
\text { chronic kidney disease }\end{array}$ & No & No & $\begin{array}{l}\text { Before } \\
\text { intuba- } \\
\text { tion }\end{array}$ & $\begin{array}{l}\text { CXR } \\
(\mathrm{PM})\end{array}$ & No & $\begin{array}{l}\text { Conserva } \\
\text {-tive }\end{array}$ & $\begin{array}{l}\text { Resol } \\
\text {-ved }\end{array}$ \\
\hline
\end{tabular}

CXR: chest X ray, chest CT: chest computed tomography scan, PM: pneumomediastinum, SE: subcutaneous emphysema, PTX: pneumothorax, ICD: intercostal intrapleural drainage, IDDM: insulin dependent diabetes mellitus, NIDDM: non-insulin dependent diabetes mellitus, DM: diabetes mellitus, N/A: information not available

There were 28 male and 6 female patients (male to female ratio 14:3). The mean age is 55 years (range $23-84$ years).

Nine patients reported to have pre-existing chronic respiratory diseases $(23 \%) ; 5$ asthmatic, 2 other chronic lung diseases, and two cases of obstructive sleep apnoea. Two patients were smokers.

Cough was among the presenting symptoms in 22 patients, while 16 patients did not experience cough (ratio 11:8) and it was not mentioned for one patient if cough was present or not.

A total of 32 patients had received oxygen therapy (82.1\%) while 7 patients did not receive any Oxygen therapy when pneumomediastinum was diagnosed $(17.9 \%)$. The risk ratio (RR) is 4.6. 14 patients had developed pneumomediastinum during or after endo-tracheal intubation; three of them sustained iatrogenic airway injury (21.4\%). The other 25 patients were not subjected to endo-tracheal intubation when they developed the condition.

Pneumomediastinum was apparent in plain chest radiographs in 10 patients $(25.6 \%)$, and was visible on chest computed tomography scan only in 29 patients (74.4\%). Figure 1 shows the appearance of pneumomediastinum in chest $\mathrm{x}$ ray and chest computed tomography (CT) scan.

Figure 2 illustrates the radiological findings in the reported patients.

Pleural drainage was not necessary in the majority of cases. Three pneumomediastinum patients had pleural and subcutaneous drainage while 4 patients with co-existent pneumothorax were treated conservatively ( $p$ value $=0.542$ ). Figure 3 demonstrates the management strategies applied to the reported patients, and Figure 4 represents the management outcome. In Figure 5 the outcomes are related to the management strategies; conservation versus intervention ( $\mathrm{p}$ value $=0.327$ ). No difference was observed in the outcome between the groups who developed the condition with or without endotracheal intubation ( $\mathrm{p}$ value $=0.646$ ).

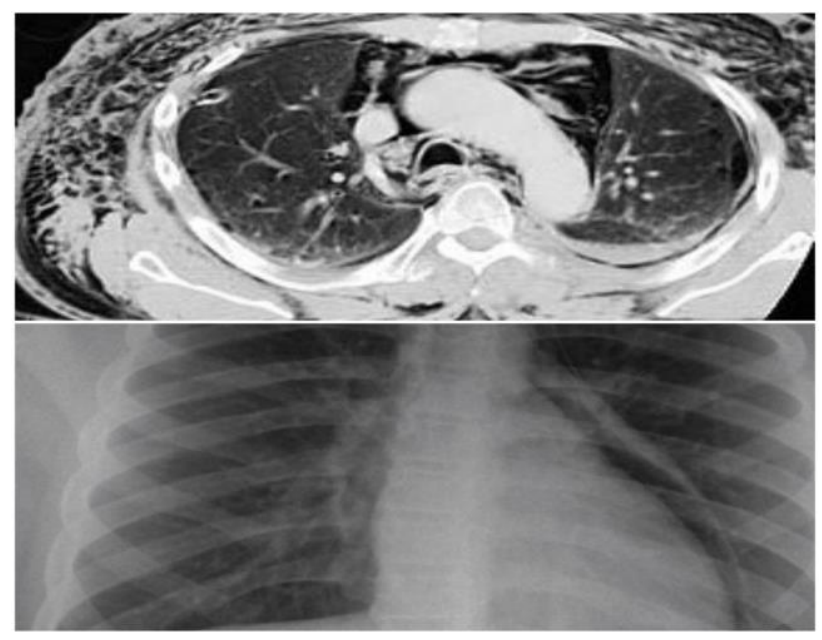

Figure 1: Appearance of pneumomediastinum in chest $x$ ray and $C T$ scan.

One patient had developed spontaneous pneumomediastinum which persisted after endo-tracheal intubation, and it persisted also in another patient who had never been intubated.

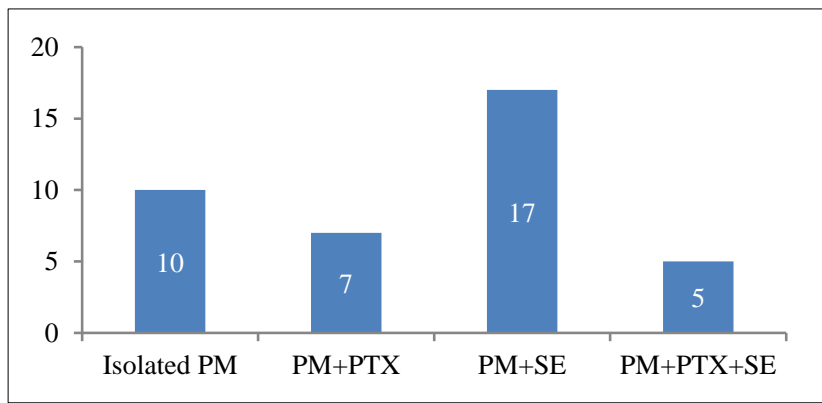

Figure 2: Radiological findings in reported patients. PM: pneumomediastinum, PTX: pneumothorax, SE: subcutaneous emphysema. 


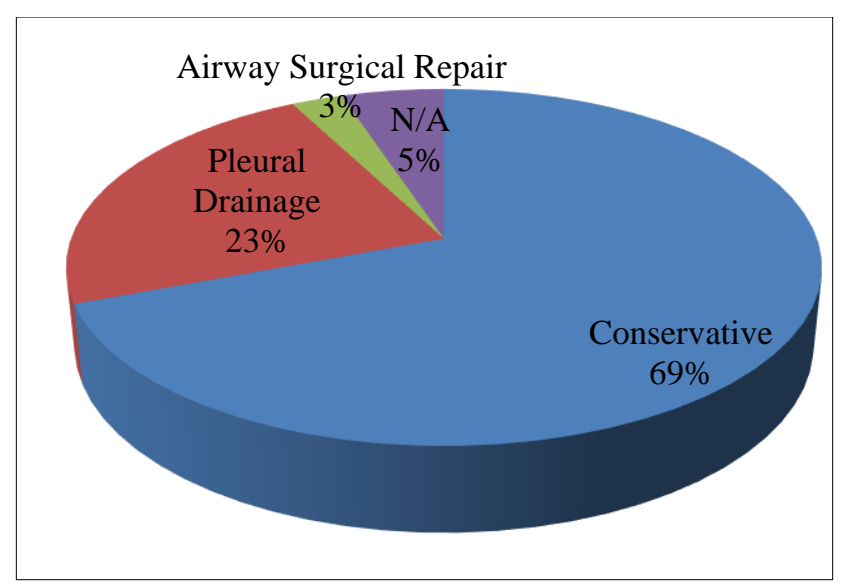

Figure 3: Management strategies applied to reported patients.

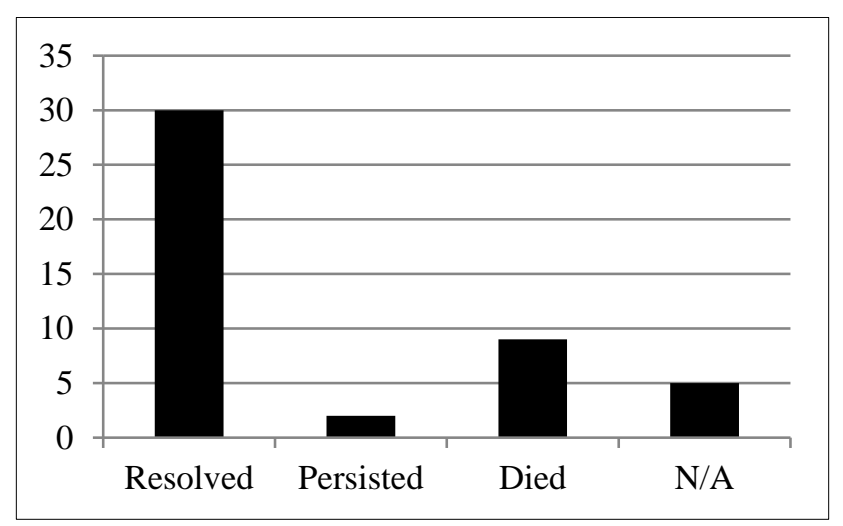

Figure 4: Management outcome.

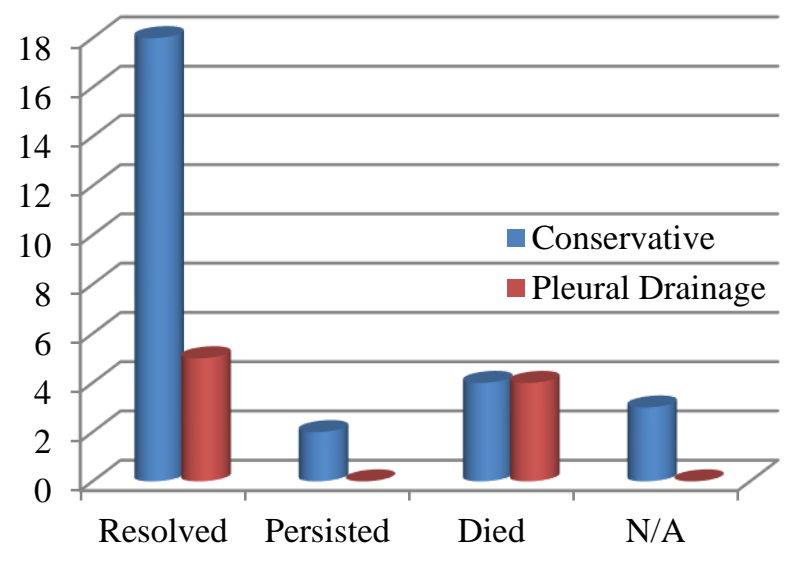

Figure 5: Outcomes related to management strategy.

\section{DISCUSSION}

Pneumomediastinum is not a known classical finding in the course of coronavirus infection. Although it may be precipitated by barotrauma; especially in a chronically diseased lung, its development without such a cause in many coronavirus infected patients had recently attracted attention.
In this review, males were affected more than females, a finding that correlates with the general incidence of the condition, although it had affected an older age group (mean age 55 years) than the average in these reported cases. $^{2}$

Pre-existing lung disease or cough did not appear to be a significant precipitating factor for pneumomediastinum development in this cohort of patients. Oxygen therapy, being invasive or non-invasive, carried a four-fold risk of developing the condition. However, this can simply reflect that the gas exchange was critically compromised in the more severely-affected lungs and thus the alveoli were more readily prone to rupture in response to minor pressure increases. What stands against this hypothesized explanation is the $12.8 \%$ of patients who developed the condition without any exposure to oxygen therapy or having cough as a symptom.

Diagnosis of the condition required advanced imaging in the form of computed tomography scanning in the majority of patients. In one patient only the clinically apparent subcutaneous emphysema had prompted imaging that had led to the diagnosis of pneumomediastinum, in spite the fact that subcutaneous emphysema is the commonest clinical sign of pneumomediastinum in other circumstances. ${ }^{2}$ Nevertheless, subcutaneous emphysema was detected radiologically in $56 \%$ of the patients. Associated pneumothorax was radiologically evident in $30 \%$ of the subjects compared to the generally reported incidence ranging from $14 \%$ to $40 \%$. $^{2,24}$

There was no statistical difference between the two management strategies in terms of outcome, and the majority of patients responded favorably to management whether conservative or interventional (pleural drainage, surgical repair of airway injury). Death incidence was evenly distributed between the two groups. Lack of autopsy-derived information makes merely attributing these fatalities, in the context of coronavirus infection, to pneumomediastinum a kind of conjecture.

Macklin and Macklin in their excellent work published in 1944 considered pneumomediastinum in the context of serious lung inflammatory process and existence of cough indicators of severity; what they called "malignant pneumomediastinum". 25 A detailed description of the disease process, pathophysiology and autopsy findings of a cohort of patients during the influenza epidemic 1918 by Torrey and Grosh led them to the believe that presence of free air around blood vessels compressing them and reducing blood movement to and from the chest thus interfering with the cardiac function, together with the effect of the air in the connective tissue preventing the lungs from collapse during expiration, are the grounds of the pneumomediastinum deleterious consequences. ${ }^{26}$

Although Chu et al reported that pneumomediastinum is a frequent complication of severe acute respiratory syndrome which carries poor prognostic indication during 
the SARS epidemic, this cannot be considered true for the coronavirus infection; both being of viral origin; based on currently available data. ${ }^{27}$

\section{CONCLUSION}

There is no enough evidence to suggest that pneumomediastinum is a natural disease process in coronavirus infection, or it is just a complication of treatment interventions. A poor prognostic value of pneumomediastinum is not supported by the currently available data. Both conservative and interventional management are successful depending on the clinical scenario and the treating doctor preference. It is worth to mention that most of these case series did not report the total number of the patient cohorts treated in their institutions in order to generate valuable statistical assumptions. Moreover, the number of un-diagnosed and un-reported cases may be quite significant to make a difference. Further well-designed observational studies of enough sample size may shed more light on the topic.

\section{Funding: No funding sources \\ Conflict of interest: None declared \\ Ethical approval: Not required}

\section{REFERENCES}

1. Zylak CM, Standen JR, Barnes GR, Zylak CJ. Pneumomediastinum Revisited, Radiographics. 2000;20(4):1043-57.

2. Kouritas VK, Papagiannopoulos K, Lazaridis G, Baka S, Mpoukovinas I, Karavasili V, et al. Pneumomediastinum. J Thorac Dis. 2015;7(1):44-9.

3. Vega JML, Gordo MLP, Tascón AD, Vélez SO. Pneumomediastinum and spontaneous pneumothorax as an extrapulmonary complication of COVID-19 disease. Emerg Radiol. 2020;1-4.

4. Zhou C, Gao C, Xie Y, Xu M. COVID-19 with spontaneous pneumomediastinum. Lancet Infect Dis. 2020;20:510.

5. Wang W, Gao R, Zheng Y, Jiang L. COVID-19 with spontaneous pneumothorax, pneumomediastinum and subcutaneous emphysema. J Travel Med. 2020;27(5):taaa062.

6. Xiang $\mathrm{C}, \mathrm{Wu}$ G. SARS-CoV-2 pneumonia with subcutaneous emphysema, mediastinal emphysema, and pneumothorax. Medicine. 2020;99(20):e20208.

7. Mohan V, Tauseen RA. Spontaneous pneumomediastinum in COVID-19. BMJ Case Rep. 2020;13:e236519.

8. Lacroix M, Graiess F, Monnier Cholley L, Arrivé L. SARS-CoV-2 pulmonary infection revealed by subcutaneous emphysema and pneumomediastinum, Intensive Care Med. 2020;46:1620-1.

9. Eperjesiova B, Hart E, Shokr M, Sinha P, Ferguson GT. Spontaneous Pneumomediastinum/Pneumo thorax in Patients With COVID-19. Cureus. 2020;12(7):e8996.
10. Abou-Arab O, Huette P, Berna P, Mahjoub Y. Tracheal trauma after difficult airway management in morbidly obese patients with COVID-19. Br J Anaesth. 2020;125(1):168-70.

11. Al-Azzawi M, Douedi S, Alshami A, Al-Saoudi G, Mikhail J. Spontaneous Subcutaneous Emphysema and Pneumomediastinum in COVID-19 Patients: An Indicator of Poor Prognosis? The American Journal of Case Reports. 2020 Jul; 21:e925557. DOI: 10.12659/ajcr.925557.

12. Collercandy N, Guillon A. Pneumomediastinum in critically ill adult with COVID-19. Med Intensiva. 2020.

13. Wegner U, Jeffery G, Abrajan O, Sampablo I, Singh C. Spontaneous Pneumomediastinum Associated With SARS-CoV-2: Infrequent Complication of the Novel Disease. Cureus. 2020;12(7):9189.

14. Goldman N, Ketheeswaran B, Wilson H. COVID-19associated pneumomediastinum. Clin Med. 2020;20(4):91-2.

15. Sun R, Liu H, Wang X. Mediastinal Emphysema, Giant Bulla, and Pneumothorax Developed during the Course of COVID-19 Pneumonia. Korean J Radiol. 2020;21(5):541-4.

16. Wang J, Su X, Zhang T, Zheng C. Spontaneous Pneumomediastinum: A Probable Unusual Complication of Coronavirus Disease 2019 (COVID19) Pneumonia. Korean J Radiol. 2020;21(5):627-8.

17. Kolani S, Nawfal H, Haloua M, Alaoui Lamrani Y, Boubbou M, Serraj M, et al. Spontaneous pneumomediastinum occurring in the SARS-COV-2 infection. ID Cases. 2020;11(21):e00806.

18. Brogna B, Bignardi E, Salvatore P, Alberigo M, Brogna C, Megliola A, et al. Unusual presentations of COVID-19 pneumonia on CT scans with spontaneous pneumomediastinum and loculated pneumothorax: A report of two cases and a review of the literature. Heart Lung. 2020.

19. Romano N, Fischetti A, Melani EF. Pneumomediastinum related to COVID-19 pneumonia. Am J Med Sci. 2020.

20. Quincho-Lopez A, Quincho-Lopez DL, HurtadoMedina FD. Case Report: Pneumothorax and Pneumomediastinum as Uncommon Complications of COVID-19 Pneumonia-Literature Review. Am J Trop Med Hygiene. 2020;103(3):1170-6.

21. Wali A, Rizzo V, Bille A, Routledge T, Chambers A. Pneumomediastinum following intubation in COVID-19 patients: a case series. Anaesthesia. 2020;75:1076-81.

22. Janseen J, Kamps MJA, Joosten TMB, Barten DG. Spontaneous Pneumomediastinum in a male adult with COVID-19 pneumonia. Am J Emerg Med. 2020.

23. Volpi S, Ali JM, Suleman A, Ahmed RN. Pneumomediastinum in COVID-19 patients: a case series of a rare complication. Eur J Cardio-Thoracic Surg. 2020;58(3):646-7.

24. Caceres M, Ali SZ, Braud R, Weiman D, Garrett HE. Spontaneus pneumomediastinum: a comparative 
study and review of the literature. Ann Thoracic Surg. 2008;86(3):962-6.

25. Macklin MT, Macklin C. Malignant interstitial emphysema of the lungs and mediastinum as an important occult complication in many respiratory diseases and other conditions: an interpretation of the clinical literature in the light of laboratory experiment. Medicine. 1944;23(4):281-358.

26. Torrey, Robert G, Lawrence C. Acute pulmonary emphysema observed during the epidemic of influenzal pneumonia at Camp Hancock, Georgia. Am J Med Sci. Philadelphia. 1919;157(2):170.
27. Chu CM, Leung YY, Hui JYH, Hung IF, Chan VL, Leung WS, et al. Spontaneous pneumomediastinum in patients with severe acute respiratory syndrome. Eur Respir J. 2004;23(6):802-4.

Cite this article as: Kanani E. Pneumomediastinum with COVID-19: a natural process or complication? A literature review. Int Surg J 2020;7:3868-3875. 